\title{
Rechtsgeschichte
}

www.rg.mpg.de

http://www.rg-rechtsgeschichte.de/rg15

Zitiervorschlag: Rechtsgeschichte Rg 15 (2009)

$\operatorname{Rg} 15_{2009}$

$175-179$

http://dx.doi.org/10.12946/rg15/175-179

\section{Gerhard Ries}

\section{Versuch ist nicht strafbar}


Überblickt man abschließend nochmals die Sachgruppen, zu denen die Stichworte im Registerband vereint sind, dann hat man als Jurist das Gefühl, nur mit den unangenehmen Seiten des Lebens (Autorität, Gebot, Herrschaft, Staat, Recht und Unrecht, Vorwerfbarkeit, Schuld, Sanktion und Strafe) zu tun zu haben. Die heiteren und schönen Seiten des Daseins - oder der Philosophie - erschließen sich erst mit den hier ebenfalls behandelten Stichworten Bohème, Gefühl, Grazie, Anmut, Humor, Kitsch und Kunst, das Schöne, Spiel und Spleen, aber auch mit Hochherzigkeit, Ironie, der Hörnerfrage und dem Krokodilschluss, mit »das Lächerliche", dem Lustprinzip oder der Seelenruhe. Noch viele Schlüsselbegriffe gibt es, nach denen der im Weinberg von Staat und Gesellschaft »normativ « arbeitende Jurist sich sehnen könnte. In seinen Gegenständen findet er all dies kaum.
Man kann ihm deshalb nur empfehlen, über das Register auch andere Stichworte des Historischen Wörterbuchs der Philosophie zu Rate zu ziehen. Mit Sicherheit findet er auf der grünen Weide jenseits der Jurisprudenz genügend Futter für seine Docta ignorantia. Mag er wie Oblomow im Bett liegen und das Wörterbuch für die Welt nehmen, um sich nicht in die Welt begeben zu müssen, mag er der Kunst des Oblivionismus huldigen (Harald Weinrich), also des planmäßigen Vergessens, um die Seele zu entlasten: In jedem Fall kann er seinem Ignoramus, ignorabimus (auch dies ein juristisches Stichwort) wirksam abhelfen, entweder durch einen raschen Griff ins Regal oder durch Installation der fabelhaften blauen Scheibe (CD-ROM), die dem Registerband beiliegt.

Michael Stolleis

\section{Versuch ist nicht strafbar*}

Die Arbeit befasst sich mit grundlegenden Fragen der Gesetzessystematik und damit implizit auch mit der Frage der »Wissenschaftlichkeit « der Gesetze Hammurapis (I 8. Jh. v. Chr.). $\mathrm{Da}$ aus dieser Zeit und auch im gesamten Zeitraum der so genannten "Keilschriftenrechte", der grob bemessen die ersten drei Jahrtausende v. Chr. umfasst, keine theoretische Literatur zum Recht - mit Ausnahme allgemeiner Reflexionen zum Wesen des Rechts - überliefert ist, bleibt dem Rechtshistoriker bei der Erforschung des geistigen Hintergrunds nur die Analyse der Quellen der Rechtspraxis. Harke stützt sich dabei ausschließlich auf normative Texte und lässt die große Fülle der urkundlichen Überlieferung ganz außer Betracht, obwohl gerade aus der Zeit um Hammurapi eine große Anzahl Vertrags- und Gerichtsurkunden überliefert ist. Auch wenn diese Texte zum Thema der Gesetzessanktionen nur von begrenztem Aussagewert sind, hätte doch auf diesen Quellentyp Bezug genommen werden sollen.

Harke kommt bei der Frage, ob die Folgen für Rechtsverstöße (so scheint der nicht weiter definierte Begriff »Sanktion « verstanden zu sein) einer begrifflichen Systematik folgen, zum Ergebnis, dass »die Verfasser des Gesetzbuchs bei der Auswahl der Rechtsfolgen nicht wahllos verfahren sind, sondern klar zwischen Schadensersatz und Strafe so differenziert und sie an

\footnotetext{
* Jan Dirk Harke, Das Sanktionensystem des Codex Hammurapi (Würzburger rechtswissenschaftliche Schriften 70), Würzburg: Ergon Verlag 2007, 96 S., ISBN 978-3-89913-574-9
} 
verschiedene Voraussetzungen gebunden haben " (55). Fahrlässigkeit und Vorsatz werden dabei als grundsätzliche Unterscheidungsmerkmale für die verschiedenen Sanktionsarten gesehen. Die Möglichkeit, dass Sanktionen allein an die Verursachung einer Schädigung geknüpft werden, scheint der Verfasser auszuschließen (vgl. etwa Io mit A. 7; 25-27; 32 mit A. 86; 55). Um diese einem Großteil der Argumentation zugrundeliegende These aufrechterhalten zu können unterstellt Harke bei Normen, die jedenfalls einen expliziten Hinweis auf Vorsatz bzw. Fahrlässigkeit nicht enthalten, das Erfordernis eines dieser Verschuldenskriterien. Das ist schon deshalb zweifelhaft, weil das Gesetz Formulierungen enthält, die die Außerachtlassung von Sorgfalt ausdrücklich zur Voraussetzung der Haftung machen: $\mathbb{5} 5$ Z. 34: abšsu iddi »er (der einen Bewässerungskanal öffnet und am Nachbargrundstück dadurch Schaden verursacht) hat seinen Arm schlaff herabhängen lassen « oder \$237, Z. 46: igi »er (der Mieter des Schiffes, der Schaden an dem Schiff verursacht) ist nachlässig “, (ähnlich $\mathbb{1 0 5}, \mathrm{Z} .47 ; \mathbb{1 2 5}, \mathrm{Z} .75$; $\$ 267$, Z. 82). Wenn man dem Gesetzgeber ein so hohes Systembewusstsein zuspricht, wie in der Arbeit angenommen, so müsste doch jedenfalls geklärt werden, weshalb bei Normen, die nur die Verursachung eines Schadens für die Sanktion voraussetzen und die Fahrlässigkeit nicht ausdrücklich ansprechen, dennoch Fahrlässigkeit erforderlich sein soll.

Ähnlich wie bei der Fahrlässigkeit geht Harke auch beim Vorsatz vor: Bei Delikten mit besonders strengen Sanktionen geht er - meist ohne jeden Anhaltspunkt im Text des gesetzlichen Tatbestandes - von Vorsatztaten aus, insbesondere bei Delikten, für die die Talion angedroht ist ( 25 f.). So wird für die Fälle der unberechtigten Verdächtigung (z.B. $\mathbb{S} \mathbb{I}$ I, 2, II) notwendiger- weise »böser Wille « vorausgesetzt, obwohl sehr wohl denkbar ist, dass der Ankläger von der Richtigkeit seiner Anklage überzeugt ist, die Beweise dann aber ein anderes Ergebnis erbringen. Als Argument führt der Verfasser an, dass »eine Talion der schädlichen Absicht stattfindet « (26). Was damit gemeint sein soll, bleibt unklar: Talion heißt Vergeltung gegen den Täter mit der Schädigung, die dem Opfer angetan worden ist. Eine schädliche Absicht kann man dem zu Unrecht Anschuldigenden aber nicht antun. Die Argumentation, die sich gegen die einleuchtenden Überlegungen von Nörr und Szlechter (26, A. $65 ; 67$ ) wendet, bleibt ganz unbefriedigend. Dasselbe gilt auch für die Behauptung, die Talionsbestimmungen der $\mathbb{S} \mathbb{S} 196 \mathrm{ff}$. setzten Vorsatz des Täters voraus (26). Harke leitet dies aus dem Kontext der Bestimmungen her. $\mathbb{2 0 6}$, der die Körperverletzung im Streit behandelt, sagt uns, dass derjenige, der im Streit einem anderen eine Wunde zufügt, nicht der Talion, sondern nur der einfachen Schadensersatzhaftung unterliegt. Versteht man das als - sinnvolle - Privilegierung des Schädigers (den Geschädigten trifft ja ein Mitverschulden), so passt diese Norm auch zur systematischen Stellung im Gesetz, wenn man von folgendem Schema ausgeht: Grundsätzlich ist die Verletzung mit der Talion auch dann zu ahnden, wenn keinerlei Schuld

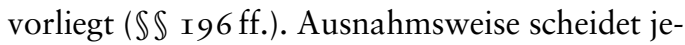
doch Talion aus, wenn die Verletzung im Streit stattgefunden hat und der Täter durch seinen Eid nachweist, dass er diese Verletzung nicht gewollt hat.

Bei der Verursachung einer Fehlgeburt durch einen Schlag gegen den Körper einer schwangeren Frau ( $\$ 209)$ nimmt Harke an, dass das Verbum mabāșum »schlagen « nur die »Vorsatzvariante « bedeuten könne (26). Wenn man sich auf sprachliche Argumente stützt, so sollte man 
jedoch zumindest die Bedeutungen eines Wortes berücksichtigen, die sich aus dem näheren textlichen Umfeld ergibt. mabūṣum ist das Wort für die Schädigung in $\$ 240, Z$. 70. Bei dieser Bestimmung geht es um die Kollision zweier Schiffe. Hier kann man nur schwerlich annehmen, dass der gesetzliche Tatbestand die »Vorsatzvariante " betrifft, der Kapitän also das andere Schiff vorsätzlich gerammt hat. Dieser Ansicht ist auch der Verfasser nicht, wenn er bei der tabellarischen Beschreibung der einzelnen Normen der Gesetze Hammurapis $\$ 240$ unter der Rubrik »fahrlässiges Delikt « führt ( $9 \circ \mathrm{f}$.). Nicht sprachlich, aber doch rechtlich verfehlt ist schließlich die Annahme, der Arzt hafte gemäß $\ 2$ I 8 als »Vorsatztäter «, wenn der Patient aufgrund der Operation stirbt oder sein Auge verliert (27). Dass der Vorsatz nach allen anerkannten Definitionen ein Wissen und Wollen des objektiven Tatbestandes voraussetzt, also hier auch des erfolglosen Ausgangs der Operation, ist dabei nicht beachtet. Eine eigene, zu der vorgelegten Interpretation passende Definition des Vorsatzes versucht der Verfasser allerdings auch nicht. Die Grundthese der Arbeit, Strafe setze Verschulden in Form von Vorsatz oder Fahrlässigkeit voraus, bleibt damit ohne brauchbare Grundlage.

Auf der Suche nach Ordnungsprinzipien geht Harke die einzelnen Normen nach dem Typus ihrer Rechtsfolgen durch ( $\mathrm{I}_{3} \mathrm{ff}$.). Dabei bespricht er knapp solche Vorschriften, die Preise und dispositives Vertragsrecht betreffen. Die Preisvorschriften bezeichnet er als bindend für Palast und Tempel; für die Allgemeinheit seien sie eher Richtwerte gewesen, in denen man eine unverbindliche soziale Zielvorgabe sehen müsse. Ob diese Behauptung haltbar ist, hätte man anhand der großen Anzahl von Vertragsurkunden jener Zeit untersuchen müssen, denn prima facie leuchtet es wenig ein, dass der Gesetzgeber einen doch erheblichen Teil seiner Normen nur als unverbindliche Empfehlung erlassen hat. Harke bemüht sich nicht um diesen Aspekt. Er unterscheidet dann aber nach dispositiven und zwingenden Normen. Letztere seien bei Normen zum Schutz wirtschaftlich benachteiligter Personen anzunehmen. Wieso aber etwa Lohnvorschriften nicht auch zu solchen sozialrechtlichen Normen gehören (I4), ist in der Arbeit nicht weiter dargelegt.

Die Ausführungen zu »sachen-, familienund erbrechtlichen Bestimmungen « (I6 ff.) sind inhaltlich zutreffend, können aber keine Argumente für die Grundfrage der Arbeit bieten, da sie nicht von Gesetzesverstößen handeln.

Bei der Sanktion der »Geldstrafen « (29 ff.) vermisst man die Frage, wem diese zu entrichten sind. Stehen sie dem Geschädigten zu, was in den von Harke nicht berücksichtigten $\mathbb{S}$ I06, Z. 66; \II2, Z. 73 und $\ 265, Z .74$ ausdrücklich gesagt wird, dann wäre der in der antiken Rechtsgeschichte etablierte Begriff der "Geldbuße « oder der »Privatstrafe« angebracht. Dass die Pflicht der Geldleistung in bestimmten Fällen als Minus zu strengeren Strafen (Todesstrafe, Verstümmelung) gesehen werden kann, ist zutreffend gesehen (29 f.). Der Verfasser führt hier als einschlägiges Beispiel noch einmal $\mathbb{2 0 6}$ an, übersieht aber, dass es dabei weder um die fahrlässige Herbeiführung der Todesfolge durch Beteiligung an einer Schlägerei geht noch hier überhaupt von einer Geldstrafe/-buße die Rede ist, sondern vom Ersatz des konkreten Schadens (Arztkosten für den überlebenden, also nicht getöteten Geschädigten) (32).

Die Überlegung zur »Kompensation durch Naturalherstellung oder Schadensersatz in Geld « (33 ff.) wird von einem unverständlichen Satz eingeleitet: Woran wird die Todesfolge geknüpft 
(an die Beteiligung an der Schlägerei oder an die Geldstrafe) und betrifft die Todesfolge den Tatbestand des $\mathbb{2 0 6}$ (dann derselbe Fehler wie Seite 32) oder dessen Rechtsfolge (dann ein erneuter Fehler)? Die Interpretation von $\mathbb{S} \mathbb{S}$ I99, 2I9, 220 (33 f.) folgt konsequent der (zweifelhaften) Annahme, es handele sich hier um Vorsatztaten.

Bei der »Nichterfüllung vertraglicher Pflichten « $(35 \mathrm{ff}$.) hält Harke den schwierigen Tatbestand des $\mathbb{S} 25$ für einen Nachweis, dass die Fahrlässigkeit eines Vertragsschuldners nur dort erwähnt wird, "wo vorsätzliches Verhalten ein Delikt bedeutete, das eine Strafe nach sich zöge«. Mit dieser Bestimmung wäre dann aber $\$ 267$ $\mathrm{zu}$ vergleichen gewesen, der in der tabellarischen Zusammenstellung zu Recht als Tatbestand einer Vertragsverletzung gesehen wird (94). Hier ist wie in $\mathbb{1} 25$ ebenfalls die Fahrlässigkeit des Vertragsschuldners (Hirten) angesprochen (Z. 82), doch ist die Schädigung hier kaum als vorsätzliches Delikt denkbar: Der Hirte hat eine Lähmung (vgl. v. Soden, Akkadisches Handwörterbuch, 857 ) der Tiere im Viehhof bewirkt; wie sollte er diese Lähmung vorsätzlich bewirken können?

Die Rechtsfolge der Verwirkung als mögliche Sanktion bei Vertragsverletzungen ist anschaulich dargestellt. Das wohl prominenteste Beispiel dafür ist das wucherische Darlehen, bei dem der wucherische Darlehensgeber weder den überhöhten Zinssatz noch die gewährte Darlehensvaluta verlangen kann $\left(\mathbb{S} \mathrm{S}_{52}\right)$. Harke behauptet allerdings, der Gesetzgeber habe mit der Verwirkung des Rückforderungsanspruchs nicht die Effektivität des Verbots gesteigert, sagt aber im folgenden Satz, dass die Verwirkung die Funktion gehabt haben könne, vor der Missachtung der Zinsgrenzen abzuschrecken. Was hier gemeint ist, bleibt mir unverständlich.
In seinem Vergleich der Gesetze Hammurapis und der Gesetze von Ešnunna stellt Harke schließlich noch Unterschiede und Gemeinsamkeiten in den Sanktionen beider Gesetze heraus ( 57 ff.). $\mathrm{Zu}$ den bedeutendsten Unterschieden gehört das Fehlen des Talionsrechts in den Gesetzen von Ešnunna (58). Beim Thema Vorsatz Fahrlässigkeit - Erfolgshaftung geht der Verfasser auf $\$ 36$ GE ein, bei dem er den Nachweis der Nachlässigkeit des Verwahrers annimmt für den dort geregelten Fall, dass das verwahrte Gut aus dem verschlossenen und nicht aufgebrochenen Haus gestohlen worden ist (58, A. I78). Näher als diese - nicht weiter begründete - These liegt hier aber die Annahme, dass in diesem Fall Vorsatz des Verwahrers in typisierter, an äußeren Merkmalen orientierter Weise anzunehmen ist. Wenig plausibel ist auch die Annahme eines Versuchsdeliktes in $\mathbb{S} \mathbb{S} 2 \mathrm{f}$. GE. Die Bestimmungen sprechen nur von einem unerlaubten Aufenthalt in einem fremden Getreidefeld bzw. Haus. Auch R. Yaron, Laws of Ešnunna, 2. Aufl., 274, den Harke als Gewährsmann für die These vom versuchten Diebstahl heranzieht, bemerkt, dass in diesen Bestimmungen nichts von "anything stolen « erwähnt sei. Man kann diese Bestimmungen als Strafnormen bezüglich einer Vorbereitung des Diebstahls ansehen, nicht aber, wie Harke meint, als Tatbestand, der einen Diebstahl unterstellt (60). Die Versuchsthese ist neben den Gründen aus dem sprachlichen Befund auch deshalb ganz unwahrscheinlich, weil keines der altorientalischen Gesetze den Versuch (nicht einmal des Mordes!) für strafbar erklärt. Offensichtlich war für die Strafbarkeit immer ein bestimmter Erfolg erforderlich. Ohne konkrete Schädigung war selbst die niedrigste Gesinnung kein strafrechtliches Unrecht.

In einem Anhang (65-96) sind sämtliche Bestimmungen der Gesetze Hammurapis tabel- 
larisch aufgeführt - unabhängig davon, ob in ihnen "Sanktionen « im Sinne des Verfassers enthalten sind. Die Logik dieser Tabelle ist dabei nicht immer klar. So wird z. B. $\$ 232$ sowohl als fahrlässiges Delikt als auch als Vertragsverletzung qualifiziert, $\mathbb{S} \mathbb{S}$ 229-23 I (fehlerhafte Erfüllung eines Vertrags zum Bau eines Hauses wie bei $\ 232$ ) werden dagegen nur als fahrlässiges Delikt bezeichnet und $\mathbb{2} 33$, der ebenfalls eine Schlechterfüllung eines Hausbauvertrages betrifft, nur als Vertragsverletzung (90). Ähnlich unzureichend ist die Qualifikation der $\mathbb{S} \mathbb{S} 2$ I 8 220 über die Arzthaftung, die weder als fahrlässiges Delikt noch als Vertragshaftung geführt wird (89). \I3 wird schlicht als »unklar « bezeichnet (67), $\mathbb{S} \$ 35-37$ führen nach Seite 69 die Verwirkung nicht - wie im Text (47: »pönaler Charakter der Sanktion «) - unter "Strafe", sondern unter »Sonstiges «. Dies sind nur einige von vielen Ungereimtheiten dieser Tabelle.

\section{Bis in den siebten Grad*}

I. Es gibt bislang wohl keine Arbeit, die sich so eingehend mit der Entwicklung der Inzestverbote beschäftigt, wie das vorliegende Werk von Ubl. Das Buch behandelt die Zeit von der Spätantike bis zum Pontifikat Alexanders II.; ein Schwerpunkt liegt dabei im frühen Mittelalter. In je nach Epoche unterschiedlichem Umfang erstreckten sich die Inzestdelikte auf Eheschließungen mit Blutsverwandten, mit Schwiegerverwandten, mit Stiefeltern sowie mit Personen, zu denen eine geistliche Verwandtschaft (Taufpaten) bestand. Für die Zwecke der Rezension sollen die verbotenen Ehen unter Blutsverwandten im Vordergrund stehen.
Die Arbeit stellt mit wenigen Ausnahmen keinen Fortschritt auf dem Gebiet der Keilschriftenrechte dar. Sie leidet nicht zuletzt darunter, dass der Verfasser nicht in der Lage ist, anhand der Originaltexte zu arbeiten. Sie birgt die Gefahr in sich, dass an den Keilschriftenrechten interessierte Wissenschaftler, die der altorientalischen Sprachen nicht mächtig sind, die Ergebnisse der Arbeit als bare Münze nehmen. Merkwürdig berührt auch, dass Harke sich weitestgehend auf ältere Sekundärliteratur bezieht und vieles der jüngeren einschlägigen Literatur, insbesondere A History of Ancient Near Eastern Law (Handbuch der Orientalistik) 2. Abt., 72 Bd., hg. von R. Westbrook, Leiden 2003 und das Reallexikon der Assyrologie und Vorderasiatischen Archäologie, nicht zur Kenntnis genommen zu haben scheint.

\section{Gerhard Ries}

Das Inzestverbot erfasste in der Spätantike maximal die Ehe zwischen Geschwisterkindern, die nach römischer Zählung im vierten Grad verwandt sind (Cousinenehe; siehe dazu 5666). Zu Beginn des fünften Jahrhunderts erweiterten gallische Konzilien die Grenze bis in den sechsten Grad römischer Zählung (hiernach war auch die Ehe mit der Cousine zweiter Ordnung verboten). Den größten Umfang erreichte das Verbot unter Papst Alexander II. Als Inzest galten Ehen zwischen Personen, die bis in den siebten Grad kanonischer Zählung verwandt waren. Das Verbot erfasste danach die Abkömmlinge von insgesamt I 28 Stammeltern (2). Da hierzu

\footnotetext{
KarL Ubl, Inzestverbot und Gesetzgebung. Die Konstruktion eines Verbrechens (300-I I00) (Millennium-Studien 20), Berlin, New York: De Gruyter 2008, VIII, 59I S., ISBN 978-3-I I-020296-O
} 